\title{
Comparison of the New Rebound Tonometer with Tonopen in Pediatric Population in the Supine Position
}

\author{
Ko Eun Kim, MD, Yung Ju Yoo, MD, Jin Wook Jeoung, MD, Ki Ho Park, MD, Dong Myung Kim, MD \\ Department of Ophthalmology, Seoul National University Hospital, Seoul National University College of Medicine, Seoul, Korea
}

\begin{abstract}
Purpose: To investigate the clinical usefulness of a new rebound tonometer, Icare ${ }^{\circledR}$ PRO (Icare PRO), in comparison with tonopen (Tonopen AVIA ${ }^{\circledR}$ ) in infants and young children in the supine position.

Methods: Sixty (12 healthy and 48 glaucoma) eyes of 30 children with less than 4 years of age were included. Intraocular pressure (IOP) measurements were conducted in the supine position using Icare PRO and tonopen by 2 blinded, independent examiners. Correlation between the tonometers was examined by generalized estimating equation. Bland-Altman plot was used to evaluate the IOP measurement agreement between the tonometers.

Results: Mean age at the time of IOP examination was $1.4 \pm 1.1$ years. Significantly good correlation was shown between the IOP measurements by Icare PRO and tonopen $(r=0.880, p<0.001)$. Bland-Altman analysis showed the mean IOP difference between Icare PRO and tonopen as 1.7 $\mathrm{mmHg}$ with $95 \%$ limits of agreement of -5.5 to $8.9 \mathrm{mmHg}$. In addition, IOP difference did not vary according to the IOP extent.

Conclusions: For pediatric subjects in the supine position, IOP measurements by Icare PRO demonstrated a good agreement with those by tonopen. Icare PRO can be a considered as a safe and reliable method for IOP measurement in pediatric patients in the supine position.
\end{abstract}

Key words: Lcare PRO rebound tonometry; Tonopen; Pediatric patient; Supine position

\section{Introduction}

Intraocular pressure (IOP) measurement plays a critical role not only for assessing the treatment outcome, but also for determining the time for surgical intervention in glaucoma. Although the Goldmann applanation tonometry (GAT) has been accepted as a gold standard of tonometry, it is unsuitable for infants or young children who are unable to cooperate slit lamp mounted applanation. ${ }^{1}$ In such patients, the tonopen tonometer has been demonstrated to assess reliable and similar IOP values compared with GAT. ${ }^{1,2}$ However, tonopen requires topical anesthetics and still, a high degree of cooperation from the child.

Received: 2015. 4. 15. Revised: 2015. 5. 20.

Accepted: 2015. 6. 15.

Corresponding Author: Jin Wook Jeoung, MD, PhD Department of Ophthalmology, Seoul National University Hospital, Seoul National University College of Medicine, 101 Daehak-ro, Jongno-gu, Seoul 110-744, Korea

Tel: +82-2-2072-2438, Fax: +82-2-741-3187

E-mail: neuroprotect@gmail.com
The rebound tonometer, another type of recently developed hand-held tonometer, has been considered to be clinically safe and suitable for pediatric population. Previous studies have reported its easiness and acceptable tolerance in infants and small children without using topical anesthesia. ${ }^{3}$ Moreover, the IOP measurements by rebound tonometer in pediatric patients with glaucoma or glaucoma suspect were reported to be similar to those by GAT and the difference between the tonometers approximated the calibration error. $^{4,5}$

The Icare ${ }^{\circledR}$ PRO (Icare PRO) is a recently developed rebound tonometer, which has a built-in inclination sensor that allows downward IOP measurement in supine patient. Although several studies have reported the accuracy of IOP measured by Icare rebound tonometer in children, limited information is available on the correlation between IOP measured with Icare PRO and tonopen (Tonopen AVIA ${ }^{\circledR}$ ), especially in the supine position. ${ }^{6-8}$ Thus, we aimed to clinically validate Icare PRO in infants and young children in the supine position, by comparing with tonopen. 


\section{Methods}

A retrospective chart review was conducted for pediatric patients who visited the Glaucoma Clinic at Seoul National University Children's Hospital between January 2012 and October 2013. This study was approved by the Institutional Review Board of Seoul National University Hospital and was conducted in accordance with all Declaration of Helsinki.

\section{Subjects}

Children less than 4 years of age and who were unable to cooperate with GAT were included in the present study. IOP was measured in the supine position after sedation with oral chloral hydrate $(0.5-1.0 \mathrm{mg} / \mathrm{kg})$ and no other systemic anesthetic agents were used. Exclusion criteria were children who were unable to undergo IOP examination because of ocular infection or corneal surface disease, those in whom chloral hydrate was medically contraindicated, those who could not remain in the supine position, and those with history of intraocular surgery.

\section{Intraocular pressure measurements}

Rebound tonometry (Icare PRO; Icare Finland Oy, Helsinki, Finland) was performed by a trained examiner (JWJ) who was masked to all the subjects' clinical information. IOP measurements were done in a subject in the supine position without topical anesthesia, as previously described. ${ }^{6,9}$ After fitting a disposable single-use probe into the apparatus, ${ }^{10}$ the tip of the probe was held at 4 to $8 \mathrm{~mm}$ distance from the cornea of the eye and perpendicular to the corneal plane by adjusting the forehead support. ${ }^{11}$ Then, 6 IOP measurements were obtained by pressing the measurement button and the probe hit the eye and bounced back. The average of 6 readings, which was displayed on the tonometer's LCD (liquid crystal display) screen was used for analysis.

Ten minutes after Icare PRO tonometry, IOP measurements by tonopen (Tonopen AVIA; Reichert Inc., Depew, NY) were performed by another examiner (YJY) who was blinded to Icare PRO measurements or other clinical information. Tonopen measurements were done with a disposable latex cover over the tip. After instillation of proparacaine hydrochloride 0.5\% (Alcaine; Alcon Laboratories, Inc., Fort Worth, TX), IOP was obtained. The average of 10 independent readings displayed on the tonopen was used in the present study.

\section{Statistical analysis}

The method of generalized estimating equations (GEE) was used to evaluate the correlation between IOP measurements with Icare PRO and tonopen with respect to possible dependent bias when including both eyes from the same patient. ${ }^{12} \mathrm{~A}$ Bland-Altman plot was used to evaluate the agreement between the different tonometers, showing IOP difference against the average IOP value of the 2 tonometers. ${ }^{13}$ In consideration of using IOP values from both eyes, a 95\% limit of agreement of a Bland-Altman plot was statistically corrected. ${ }^{14}$ Additionally,

Table 1. Baseline characteristics of included subjects (60 eyes of 30 subjects).

\begin{tabular}{lc}
\hline Variables & \\
\hline Age (year) & $1.4 \pm 1.1$ (range 0.03-3.90) \\
Male (n, \%) & 19 (63.3) \\
IOP measured with Tonopen (mmHg) & $20.5 \pm 7.1$ (range 8.1-38.9) \\
IOP measured with Icare PRO (mmHg) & $22.2 \pm 7.8$ (range 12.0-46.0) \\
IOP difference between Icare PRO and Tonopen (mmHg) & $1.7 \pm 3.7(-7.6-10.7)$ \\
Absolute IOP difference between Icare PRO and Tonopen (mmHg) & $3.1 \pm 2.6(0.03-10.7)$ \\
Diagnosis (n, \%) & $12(20.0)$ \\
$\quad$ Healthy eyes & $2(3.3)$ \\
Eyes with secondary glaucoma & $46(76.7)$ \\
Eyes with primary congenital glaucoma & \\
\hline
\end{tabular}

IOP = intraocular pressure; Icare PRO = Icare PRO rebound tonometer.

Data are otherwise presented as mean \pm SD. 
with a modification of the Bland-Altman plot, the measurement difference between Icare PRO and tonopen was plotted against the IOP measured by tonopen. All of the statistical analyses were performed using SPSS version 21.0 software (IBM Corp, Armonk, NY). Statistical significance was set as $p$ $<0.05$.

\section{Results}

A total of 60 eyes of 30 pediatric subjects with the mean age of $1.4 \pm 1.1$ years were included. Their baseline characteristics are summarized in Table 1 . There were 12 healthy eyes, 2 eyes with secondary glaucoma, 46 eyes with primary congenital glaucoma. The mean IOP readings by Icare PRO and tonopen were $22.2 \pm 7.8 \mathrm{mmHg}$ and $20.5 \pm 7.1 \mathrm{mmHg}$, respectively.

IOP measurements by Icare PRO demonstrated good correlations with those by tonopen $(R=0.880, p<0.001$; Fig. 1$)$. Mean IOP difference between the tonometers was $1.7 \pm 3.7 \mathrm{mmHg}$, but the absolute IOP difference was $3.1 \pm 2.6 \mathrm{mmHg}$. IOP measurements by Icare PRO were mostly higher than those by tonopen, as in $71.7 \%(n=43)$ of 60 eyes. In addition, IOP

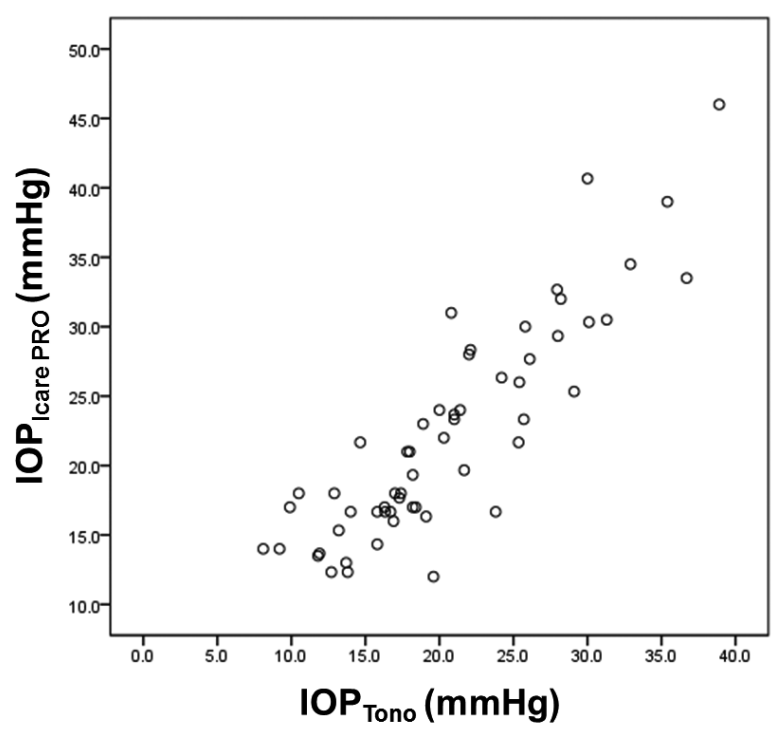

Figure 1. Scatterplots showing a significantly good correlation between intraocular pressure measurements by Icare PRO and Tonopen AVIA ( $r=0.880, p<0.001)$. IOP = intraocular pressure; $I O P_{\text {Icare PRO }}=$ IOP measured by Icare PRO rebound tonometer; IOPTono $=$ IOP measured by tonopen AVIA. measurement difference between the two tonometers was less than $2 \mathrm{mmHg}$ in $41.7 \%(\mathrm{n}=25)$ of eyes and $3 \mathrm{mmHg}$ in $56.7 \%$ $(\mathrm{n}=34)$ of eyes.

The Bland-Altman plot presenting the distribution of IOP difference between Icare PRO and tonopen is shown in Figure 2. The mean IOP difference was $1.7 \mathrm{mmHg}$ with $95 \%$ limits of agreement of -5.5 to $8.9 \mathrm{mmHg}$. Both IOP readings showed a reasonable agreement and the difference in IOP measurements between the instruments was not associated with the degree of IOP. The modified Bland-Altman plot also demonstrated that IOP difference between methods showed no correlation with IOP measured by tonopen $(r=0.080, p=0.554$; Fig. 3$)$.

\section{Discussion}

The present study found that IOP measurements by Icare PRO were in good agreement with those of tonopen in pediatric population when performed in the supine position. Although measurements by Icare PRO were mostly higher than those by tonopen, the IOP difference between the tonometries was small and showed good correlation with each other. More

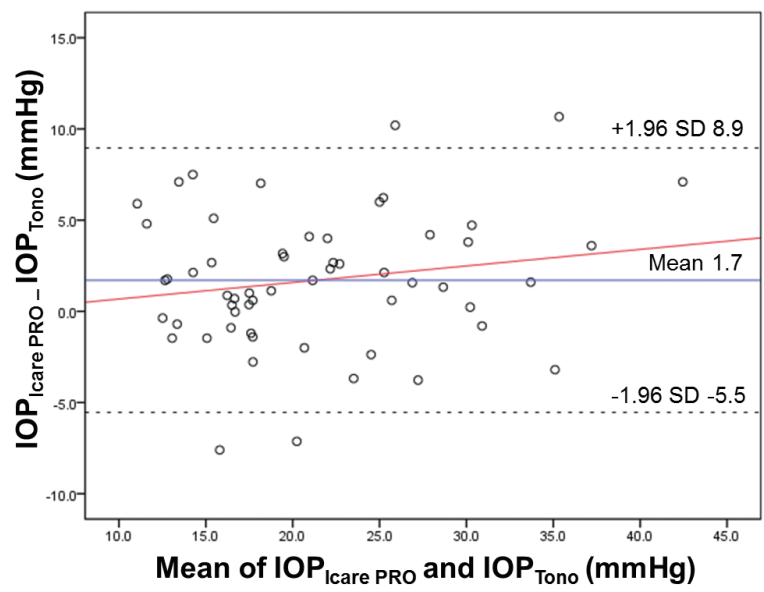

Figure 2. Bland-Altman plot presenting the distribution of intraocular pressure (IOP) measurement difference between Icare PRO and Tonopen AVIA. The $Y$-axis represents the IOP difference between the 2 tonometers and the $X$-axis represents the mean of both IOP readings. The mean IOP difference was $1.7 \mathrm{mmHg}$ with $95 \%$ limits of agreement of -5.5 to $8.9 \mathrm{mmHg}$. The regression line in red color showed that the IOP difference was not associated with the degree of $\operatorname{IOP}\left(Y=-0.229+0.091^{*} X, p=0.188\right)$. $I O P_{\text {Icare PRO }}=I O P$ measured by Icare PRO rebound tonometer; IOPTono $=$ IOP measured by tonopen AVIA. 


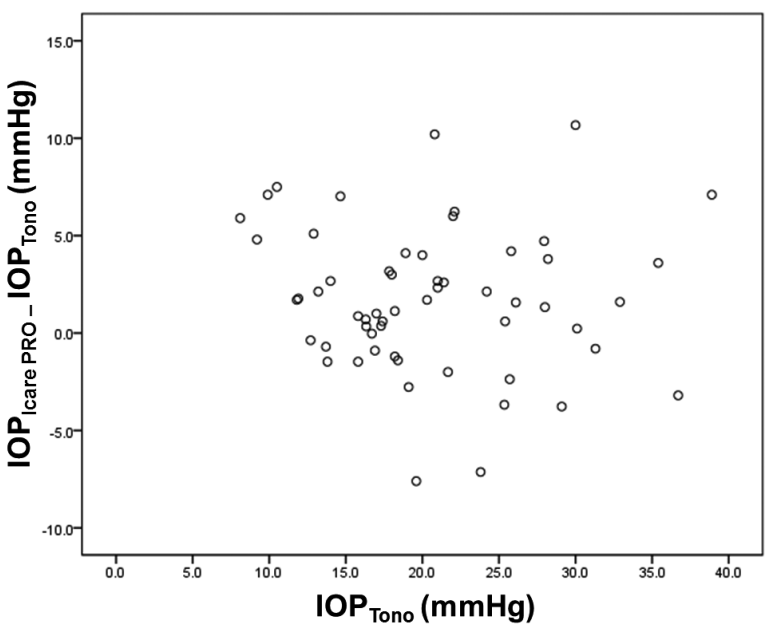

Figure 3. Modified Bland-Altman plot presenting the distribution of intraocular pressure (IOP) differences between Icare PRO rebound tonometer and tonopen against IOP measured with tonopen. The $Y$-axis represents the IOP difference between the 2 tonometers and the $X$-axis represents the IOP measurement by tonopen. The IOP difference did not vary with the IOP measured by tonopen $\left(r=0.080, Y=2.561-0.042{ }^{*} X, p=0.554\right)$. IOP Icare ${ }_{\text {PRO }}=$ IOP measured by Icare PRO rebound tonometer; $I O P_{\text {Tono }}=$ IOP measured by tonopen AVIA.

importantly, the difference in IOP measurements was not affected by the extent of IOP.

Previous studies have reported Icare rebound tonometer to be comparable to other tonometeris, ${ }^{3,5-7,9,11,15-17}$ but different results on the agreement with the tonopen measurements have been reported. ${ }^{18,19}$ Nakamura et al. ${ }^{19}$ reported very small mean IOP difference between Icare rebound tonometer and tonopen almost equal to $0 \mathrm{mmHg}$, which was smaller than our results. They also reported that Icare rebound tonometer overestimated tonopen at higher IOP and underestimated them at lower IOP, contrary to our results showing no association with IOP extent. Such discrepancy might be due to the difference in the patients' characteristics (e.g. different distribution of corneal biomechanical properties, patient age, IOP range, proportion of patients with high or low IOP) and the type of tonopen (e.g. Tonopen XL versus Tonopen AVIA).

In addition to IOP agreement between tonometers, studies have been conducted to determine the influence of position on the Icare rebound tonometry. Jablonski et al. ${ }^{7}$ reported that the mean IOP in 99 patients with glaucoma or glau- coma suspects by Icare PRO was $17.7 \pm 8.0 \mathrm{mmHg}$ in the upright position and $19.2 \pm 6.4$ in the supine position. They concluded that Icare PRO showed a good correlation and agreement with applanation and dynamic contour tonometry independent of patient's position. Nakakura et al. ${ }^{20}$ reported the mean difference between Icare and Tonopen XL as $1.47 \pm 3.52 \mathrm{mmHg}$ in the supine position, showing a relatively good agreement between them. In addition, Schweier et al. ${ }^{21}$ reported that the IOP difference obtained in different positions was lower in Icare PRO than in Tonopen AVIA. Our results also demonstrated the usefulness of Icare PRO and its good correlation with tonopen for pediatric population in the supine position. Given together, Icare rebound tonometry can obtain IOP measurements at clinically acceptable levels in diverse population independent of the position.

Our study has several limitations. First, several studies have reported that biomechanical properties of the cornea including central corneal thickness (CCT) could affect measurements by rebound tonometer. ${ }^{16,19,22}$ Due to difficulty in performing pachymetry in the majority of our pediatric subjects, the effect of CCT on IOP measurements could not have been obtained in this study. If such effects actually exist, however, they should have influenced both tonometries similarly, resulting in subtle change in the measurement difference. Second, only 1 examiner per each instrument evaluated the IOP measurements. However, we used an average value of successful and independent measurements from each device. Moreover, good inter- and intravariability of Icare PRO and Tonopen AVIA have been confirmed in other studies. ${ }^{6,15}$ Lastly, GAT is still currently accepted as a gold standard for IOP measurement, but our patients were limited to those who are unable to perform such measurement. Thus, we could only compare Icare PRO with tonopen. Further studies comparing Icare PRO, tonopen, and hand-held (Perkins) applanation tonometer in pediatric population in the supine position would provide more information on the reliability of Icare PRO.

In conclusion, although IOP measurements by Icare PRO were slightly higher than those by tonopen, the difference was insignificant and they showed a good correlation with 
each other, suggesting Icare PRO to be a reliable method for pediatric patients. Our results support the potential use of Icare PRO as a safe, quick, and reliable method for IOP measurements in pediatric population even in the supine position.

\section{References}

1. Bordon AF, Katsumi O, Hirose T. Tonometry in pediatric patients: a comparative study among Tono-pen, Perkins, and Schiotz tonometers. J Pediatr Ophthalmol Strabismus 1995;32:373-7.

2. Minckler DS, Bearveldt G, Heuer DK, Quillen-Thomas B, Walonker AF, Weiner J. Clinical evaluation of the Oculab Tono-Pen. Am J Ophthalmol 1987;104:168-73.

3. Lundvall A, Svedberg H, Chen E. Application of the ICare rebound tonometer in healthy infants. J Glaucoma 2011;20:7-9.

4. Flemmons MS, Hsiao YC, Dzau J, et al. Home tonometry for management of pediatric glaucoma. Am J Ophthalmol 2011;152:470-8 e2.

5. Gandhi NG, Prakalapakorn SG, El-Dairi MA, et al. Icare ONE rebound versus Goldmann applanation tonometry in children with known or suspected glaucoma. Am J Ophthalmol 2012;154:843-9 e1.

6. Nakakura S, Mori E, Yamamoto M, et al. Intradevice and Interdevice Agreement Between a Rebound Tonometer, Icare PRO, and the Tonopen XL and Kowa Hand-held Applanation Tonometer When Used in the Sitting and Supine Position. J Glaucoma 2013. [Epub ahead of print]

7. Jablonski KS, Rosentreter A, Gaki S, et al. Clinical use of a new position-independent rebound tonometer. J Glaucoma 2013;22:763-7.

8. Schild AM, Rosentreter A, Hermann MM, et al. [Comparison of Rebound tonometry versus Perkins tonometry in the supine glaucoma patient]. Klin Monbl Augenheilkd 2011;228:125-9.

9. Garcia-Resua C, Gonzalez-Meijome JM, Gilino J, YebraPimentel E. Accuracy of the new ICare rebound tonometer vs. other portable tonometers in healthy eyes. Optom Vis Sci 2006;83:102-7.

10. Kontiola AI. A new induction-based impact method for measuring intraocular pressure. Acta Ophthalmol Scand 2000;78:142-5

11. Muttuvelu DV, Baggesen K, Ehlers N. Precision and accuracy of the ICare tonometer - Peripheral and central IOP measurements by rebound tonometry. Acta Ophthalmol 2012;90:322-6.

12. Zeger SL, Liang KY. Longitudinal data analysis for discrete and continuous outcomes. Biometrics 1986;42:12130.

13. Bland JM, Altman DG. Statistical methods for assessing agreement between two methods of clinical measurement. Lancet 1986;1:307-10.

14. Bland JM, Altman DG. Measuring agreement in method comparison studies. Stat Methods Med Res 1999;8:13560.

15. Kim KN, Jeoung JW, Park KH, et al. Comparison of the new rebound tonometer with Goldmann applanation tonometer in a clinical setting. Acta Ophthalmol 2013;91:e392-6.

16. Brusini P, Salvetat ML, Zeppieri M, et al. Comparison of ICare tonometer with Goldmann applanation tonometer in glaucoma patients. J Glaucoma 2006;15:213-7.

17. Flemmons MS, Hsiao YC, Dzau J, et al. Icare rebound tonometry in children with known and suspected glaucoma. J AAPOS 2011;15:153-7.

18. Eisenberg DL, Sherman BG, McKeown CA, Schuman JS. Tonometry in adults and children. A manometric evaluation of pneumatonometry, applanation, and TonoPen in vitro and in vivo. Ophthalmology 1998;105:1173-81.

19. Nakamura M, Darhad U, Tatsumi Y, et al. Agreement of rebound tonometer in measuring intraocular pressure with three types of applanation tonometers. Am J Ophthalmol 2006;142:332-4.

20. Nakakura S, Mori E, Yamamoto M, et al. Intraocular pressure of supine patients using four portable tonometers. Optom Vis Sci 2013;90:700-6.

21. Schweier C, Hanson JV, Funk J, Toteberg-Harms M. Repeatability of intraocular pressure measurements with Icare PRO rebound, Tono-Pen AVIA, and Goldmann tonometers in sitting and reclining positions. BMC Ophthalmol 2013;13:44.

22. Pakrou N, Gray T, Mills R, et al. Clinical comparison of the Icare tonometer and Goldmann applanation tonometry. J Glaucoma 2008;17:43-7. 